\title{
DIÁLOGO DO PRÍNCIPE E NOBILÍSSIMO JOVEM PEPINO COM O PROFESSOR ALBINO
}

\author{
Artur Costrino* \\ Recebido em: 14/09/2020 \\ Aprovado em: 12/12/2020
}

\begin{abstract}
RESUMO: Apresento aqui nova tradução completa e anotada em português do texto Disputatio regalis et nobilissimi iuvenis Pippini cum Albino scholastico. Esse importante texto de caráter didático é composto quase que inteiramente a partir de outros textos anteriores que circulavam nos domínios carolíngios e evidencia o processo de composição de Alcuíno de Iorque, baseado, senão na emulação, certamente na prática da coleção e mistura de exemplos passados. A tradução acompanha breve introdução e notas que explicitam as respostas dos enigmas mais difíceis.
\end{abstract}

PALAVRAS-CHAVE: Alcuíno; diálogo; educação; renascimento Carolíngio.

DIALOGUE BETWEEN THE PRINCE AND THE MOST NOBLE YOUNG PIPPIN WITH PROFESSOR ALBINUS

\begin{abstract}
I present here a new and complete translation in Portuguese of the text Disputatio regalis et nobilissimi iuvenis Pippini cum Albino scholastico. This important educational text is composed almost entirely from other previous texts that circulated in the Carolingian domains and shows the process of composing of Alcuin of York, based, if not on emulation, certainly on the practice of the collection and mixture of past examples. The translation accompanies a brief introduction and notes explaining the answers to the most difficult puzzles.
\end{abstract}

KEYWORDS: Alcuin; dialogue; education; Carolingian Renaissance. 
Artur Costrino

\section{INTRODUÇÃo}

lcuíno de Iorque (Flaccus Albinus Alcuinus, mas também conhecido por seus nomes anglo-saxões, Ealhwine, por exemplo), nascido por volta do ano 735 e morto em 804 da nossa era, foi um dos pensadores, poetas e executores mais importantes do chamado "Renascimento Carolíngio" (Bullough, 2004). Sob o domínio de Carlos Magno, Alcuíno exerceu incomparável influência como principal conselheiro do rei/imperador e, principalmente, como um dos principais professores da escola palatina, encarregado da educação não só dos filhos da família real como também do projeto de letramento de todo o império. ${ }^{1}$

O texto a seguir foi escrito provavelmente na década 790, enquanto Alcuíno trabalhava no palácio em Aachen. Nesse período, antes de sua nomeação como abade de São Martinho em Tours em 796, teria escrito sua obra, a maioria composta de diálogos, dedicada às artes liberais e à educação. ${ }^{2}$

Como era frequente em Alcuíno e seus contemporâneos, o engenho do autor não estava na capacidade de criar algo "original", mas em usar, copiar e combinar textos mais antigos de modo a reinseri-los em debates ou, como era provavelmente o caso, em sala de aula. De fato, o diálogo apresentado aqui, embora curto, é uma mistura de diferentes textos antigos, de gêneros distintos.

O diálogo (de certa forma epistolar, como podemos ver pela última linha) de Alcuíno é uma mistura de diálogo sapiencial e enigmas. Dois gêneros que já tinham uma longa história, separadamente, desde o final da Antiguidade e que Alcuíno combina e condensa em um único diálogo. ${ }^{3}$ Este é uma conversa entre o professor Alcuíno, denominado "Albino" no texto, e Pepino, filho de Carlos Magno. Evidentemente, a veracidade da conversa não é verificável ou importante para o gênero. Alcuíno usa da presença da personagem da família real para elevar a importância e acesso de seu texto, como já fizera em seus outros diálogos em que o interlocutor é o próprio rei Carlos Magno.

Para a tradução, baseei-me na edição da Patrologia Latina (1844). As notas, que concernem aos enigmas mais obscuros para o público contemporâneo, foram feitas com base em outros textos que já se dedicaram ao estudo desta obra didática do professor de Iorque, como Bayless (in Halsall, 2004), Beeson e Orchard.

\footnotetext{
${ }^{1}$ Para maiores informações sobre o a Renascença Carolíngia e o papel de Alcuíno nela, v. Brown, 1994.

${ }^{2}$ Para uma maior discussão sobre a data de composição dos diálogos didáticos, v. Costrino, 2016, p. $15-27$.

${ }^{3}$ A primeira parte deste diálogo de Alcuíno segue, de forma bem próxima, o diálogo Altercatio Hadriani Augusti et Epicteti Philosophi, e também o diálogo Disputatio Hadriani cum secundo philosopho. Já na parte final, em que se encontram os enigmas, Alcuíno os busca nos Aenigmata de Sifônio, nos Aenigmata de Adelmo e na coletânea de Pseudo-Beda, dentre outros textos, incertos. A forma quase cristalizada de como começam os enigmas, a saber: "Vidi (Eu vi)" reflete não apenas a tradição latina, mas, principalmente, a forma encontrada com muita frequência no livro de Exeter, escrito em anglo-saxão: "Ic (ge)seah". Anglo-saxão que é a língua mãe de Alcuíno. Para maiores informações sobre, v. as obras de Bayless (2004), Orchard (2018) e Bitterli (2009).
} 


\section{Pippini Regalis et Nobilissimi Juvenis Disputatio cum Albino Scholastico}

Pippinus. Quid est littera? - Albinus. Custos historiae.

P. Quid est verbum? - A. Proditor animi.

P. Quis generat verbum? - A. Lingua.

P. Quid est lingua? - A. Flagellum aeris.

P. Quid est aer? - A. Custodia vitae.

P. Quid est vita? - A. Beatorum laetitia, miserorum moestitia, exspectatio mortis.

P. Quid est mors? - A. Inevitabilis eventus, incerta peregrinatio, lacrymae viventium, testamenti firmamentum, latro hominis.

P. Quid est homo? - A. Mancipium mortis, transiens viator, loci hospes.

P. Cui similis est homo? - A. Pomo.

P. Quomodo positus est homo? - A. Ut lucerna in vento.

P. Ubi est positus? - A. Intra sex parietes.

P. Quos? - A. Supra, subtus; ante, retro; dextra laevaque.

P. Quot modis variabilis est? - A. Sex.

P. Quibus? - A. Esurie et saturitate; requie et labore; vigiliis et somno.

P. Quid est somnus? - A. Mortis imago.

P. Quid est libertas hominis? - A. Innocentia.

P. Quid est caput? - A. Culmen corporis.

P. Quid est corpus? - A. Domicilium animae.

P. Quid sunt comae? - A. Vestes capitis.

P. Quid est barba? - A. Sexus discretio, honor aetatis.

\section{Diálogo do príncipe e nobilíssimo jovem Pepino com o professor Albino}

Pepino. O que é a letra? - Albino. Guardiã da História.

P. O que é a palavra? - A. Reveladora da alma.

P. O que cria a palavra? - A. A língua.

P. O que é a língua? - A. Flagelo do ar.

P. O que é o ar? - A. Guarda da vida.

P. O que é a vida? - A. A alegria dos contentes, tristeza dos infelizes, expectativa da morte.

P. O que é a morte? - A. O acontecimento inevitável, a peregrinação incerta, as lágrimas dos vivos, a fundação de um testamento, ladrão do homem.

P. O que é o homem? - A. O escravo da morte, um viajante que passa, um hóspede da casa.

P. Com o que se parece o homem? - A. Com uma árvore frutífera.

P. De que modo o homem foi colocado? - A. Como uma lanterna no vento.

P. Onde foi colocado? - A. Entre seis paredes.

P. Quais? - A. Em cima, embaixo; diante, atrás; direita, esquerda.

P. De quantos modos pode variar? - A. Seis.

P. Quais? - A. Da fome e da saciedade; do descanso e do trabalho; da vigília e do sono.

P. O que é o sono? - A. A aparência da morte.

P. O que é a liberdade do homem? - A. A inocência.

P. O que é a cabeça? - A. O topo do corpo.

P. O que é o corpo? - A. Domicílio da alma.

P. O que são os cabelos? - A. As roupas da cabeça.

P. O que é barba? - A. Distinção do sexo, adorno da idade. 
P. Quid est cerebrum? - A. Servator memoriae.

P. Quid sunt oculi? - A. Duces corporis, vasa luminis, animi indices.

P. Quid sunt nares? - A. Adductio odorum.

P. Quid sunt aures? - A. Collatores sonorum.

P. Quid est frons? - A. Imago animi.

P. Quid est os? - A. Nutritor corporis.

P. Quid sunt dentes? - A. Mola morsorum.

P. Quid sunt labia? - A. Valvae oris.

P. Quid est gula? - A. Devorator cibi.

P. Quid manus? - A. Operarii corporis.

P. Quid sunt digiti? - A. Chordarum plectra.

P. Quid est pulmo? - A. Servator aeris.

P. Quid est cor? - A. Receptaculum vitae.

P. Quid est jecur? - A. Custodia caloris.

P. Quid est fel? - A. Suscitatio iracundiae.

P. Quid est splenis? - A. Risus et laetitiae capax.

P. Quid est stomachus? - A. Ciborum coctor.

P. Quid est venter? - A. Custos fragilium.

P. Quid sunt ossa? - A. Fortitudo corporis.

P. Quid sunt coxae? - A. Epistylia columnarum.

P. Quid sunt crura? - A. Columnae corporis.

P. Quid sunt pedes? - A. Mobile fundamentum.
P. O que é o cérebro? - A. Conservador da memória.

P. O que são os olhos? - A. Os comandantes do corpo, os recipientes da luz, os indicadores da alma.

P. O que são as narinas? - A. Condutor dos odores.

P. O que são os ouvidos? - A. Coletores dos sons.

P. O que é a face? - A. Aparência da alma.

P. O que é a boca? - A. Nutriz do corpo.

P. O que são os dentes? - A. Moinhos das mordidas.

P. O que são os lábios? - A. Entrada da boca.

P. O que é a garganta? - A. Engolidor da comida.

P. O que são as mãos? - A. Operários do corpo.

P. O que são os dedos? - A. Palhetas das cordas.

P. O que é o pulmão? - A. Conservador do ar.

P. O que é o coração? - A. Receptáculo da vida.

P. O que é o fígado? - A. Guarda do calor.

P. O que é o fel? - A. Incitação da ira.

P. O que é o baço? - A. O que segura o riso e a alegria.

P. O que é o estômago? - A. O cozinheiro da comida.

P. O que é o ventre? - A. O guardião das migalhas.

P. O que são os ossos? - A. A fortaleza do corpo.

P. O que é o quadril? - A. O suporte da coluna.

P. O que são as pernas? - A. As colunas do corpo.

P. O que são os pés? - A. Alicerce móvel. 
P. Quid est sanguis? - A. Humor venarum, vitae alimentum.

P. Quid sunt venae? - A. Fontes carnis.

P. Quid est coelum? - A. Sphaera volubilis, culmen immensum.

P. Quid est lux? - A. Facies omnium rerum.

P. Quid est dies? - A. Incitamentum laboris.

P. Quid est sol? - A. Splendor orbis, coeli pulchritudo, naturae gratia, honor diei, horarum distributor.

P. Quid est luna? - A. Oculus noctis, roris larga, praesaga tempestatum.

P. Quid sunt stellae? - A. Pictura culminis, nautarum gubernatores, noctis decor.

P. Quid est pluvia? - A. Conceptio terrae, frugum genitrix.

P. Quid est nebula? - A. Nox in die, labor oculorum.

P. Quid est ventus? - A. Aeris perturbatio, mobilitas aquarum, siccitas terrae.

P. Quid est terra? - A. Mater crescentium, nutrix viventium, cellarium vitae, devoratrix omnium.

P. Quid est mare? - A. Audaciae via, limes terrae, divisor regionum, hospitium fluviorum, fons imbrium, refugium in periculis, gratia in voluptatibus.

P. Quid sunt flumina? - A. Cursus indeficiens, refectio solis, irrigatio terrae.

P. Quid est aqua? - A. Subsidium vitae, ablutio sordium.

P. Quid est ignis? - A. Calor nimius, fotus nascentium, maturitas frugum.

P. Quid est frigus? - A. Febricitas membrorum.
P. O que é o sangue? - A. O fluido das veias, alimento da vida.

P. O que são as veias? - A. As fontes da carne.

P. O que é o céu? - A. Esfera circulante, cume imensurável.

P. O que é a luz? - A. A face de todas as coisas.

P. O que é o dia? - A. Estímulo ao trabalho.

P. O que é o sol? - A. Esplendor do mundo, beleza do céu, graça da natureza, adorno do dia, distribuidor das horas.

P. O que é a lua? - A. Olho da noite, cheia de orvalho, profetiza da tempestade.

P. O que são as estrelas? - A. Pintura das alturas, capitães dos marinheiros, decoração da noite.

P. O que é a chuva? - A. Fertilizadora da terra, genitora das searas.

P. O que é a nuvem? - A. Noite durante o dia, trabalho para os olhos.

P. O que é o vento? - A. Perturbação do ar, o movimento das águas, secura da terra.

P. O que é a terra? - A. A mãe das coisas que crescem, nutriz dos viventes, celário da vida, devoradora de tudo.

P. O que é o mar? - A. O caminho da audácia, os limites da terra, divisor das regiões, pousada dos rios, fonte das garoas, refúgio nos perigos, graça nos prazeres.

P. O que são os rios? - A. Curso inacabável, descanso do sol, irrigação da terra.

P. O que é a água? - A. Subsídio da vida, limpadora das manchas.

P. O que é o fogo? - A. Calor excessivo, aquecimento dos recém-nascidos, o maduro dos frutos.

P. O que é o frio? - A. O estremecer dos membros. 
P. Quid est gelu? - A. Persecutio herbarum, perditio foliorum, vinculum terrae, fons aquarum.

P. Quid est nix? - A. Aqua sicca.

P. Quid est hiems? - A. Aestatis exsul.

P. Quid est ver? - A. Pictor terrae.

P. Quid est aestas? - A. Revestio terrae, maturitio frugum.

P. Quid est autumnus? - A. Horreum anni.

P. Quid est annus? - A. Quadriga mundi.

P. Quis ducit? - A. Nox et dies, frigus et calor.

P. Quis est auriga ejus? - A. Sol et luna.

P. Quot habet palatia? - A. Duodecim.

P. Qui sunt praetores palatiorum? - A. Aries, Taurus, Gemini, Cancer, Leo, Virgo, Libra, Scorpius, Arcitenens, Capricornus, Aquarius, Pisces.

P. Quot dies habitat in unoquoque palatio? - A. Sol XXX dies et decem semis horas. Luna duos dies et octo horas, et bisse unius horae.

P. Magister, timeo altum ire. - A. Quis te duxit in altum?

P. Curiositas. - A. Si times, descendam. Sequar quocunque ieris.

P. Si scirem quid esset navis, praepararem tibi, ut venires ad me. - A. Navis est domus erratica, ubilibet hospitium, viator sine vestigiis, vicina arenae.

P. Quid est arena? - A. Murus terrae.

P. Quid est herba? - A. Vestis terrae.

P. Quid sunt olera? - A. Amici medicorum, laus coquorum.

P. Quid est, quod amara dulcia facit? - A. Fames.

P. Quid est, quod hominem non lassum facit? A. Lucrum.
P. O que é o gelo? - A. Perseguidor das plantas, destruidor das folhas, laço das terras, fonte das águas.

P. O que é a neve? - A. Água seca.

P. O que é o inverno? - A. Exílio do verão.

P. O que é a primavera? - A. Pintora da terra.

P. O que é o verão? - A. Revestimento da terra, madureza dos frutos.

P. O que é o outono? - A. Armazém do ano.

P. O que é o ano? - A. A quadriga do mundo.

P. Quem a conduz? - A. A noite e o dia, o frio e o calor.

P. Quem é o cocheiro dela? - A. O sol e a lua.

P. Tem quantos palácios? - A. Doze.

P. Quem são os chefes dos palácios? - A. Áries, Touro, Gêmeos, Câncer, Leão, Virgem, Libra, Escorpião, Sagitário, Capricórnio, Aquário e Peixes.

P. Quanto dias habita em cada um dos palácios? - A. O sol habita trinta dias e dez meias-horas; a lua, dois dias, seis horas e dois terços de uma hora.

P. Professor, tenho medo subir muito alto. -

A. Quem o elevou muito alto?

P. A curiosidade. - A. Se você tem medo, desceremos. Eu o seguirei aonde quer que você vá.

P. Se eu soubesse o que é um barco, eu faria um para que você viesse até mim. $-\mathbf{A}$. Barco é uma casa errante, um refúgio em qualquer lugar, viajante sem pegadas, vizinho da areia.

P. O que é a areia? - A. A parede da terra.

P. O que é a relva? - A. A roupa da terra.

P. O que são os vegetais? - A. Amigos dos médicos, louvor dos cozinheiros.

P. O que é que torna o amargo doce? - A. A fome.

P. O que é que não torna o homem preguiçoso? - A. O lucro. 
P. Quid est vigilanti somnus? - A. Spes.

P. Quid est spes? - A. Refrigerium laboris, dubius eventus.

P. Quid est amicitia? - A. Aequalitas animorum.

P. Quid est fides? - A. Ignotae rei et mirandae certitudo.

P. Quid est mirum? - A. Nuper vidi hominem stantem, mortuum ambulantem, qui nunquam fuit.

P. Quomodo potest esse, pande mihi? - A. Imago in aqua.

P. Cur hoc non intellexi per me, dum toties vidi hunc ipsum hominem? - A. Quia bonae indolis es juvenis et naturalis ingenii, proponam tibi quaedam alia mira; tenta, si per teipsum possis conjicere illa.

P. Faciam; tamen ita, si secus, quam est, dicam, corrigas me. - A. Faciam, ut vis. Quidam ignotus mecum sine lingua et voce locutus est, qui nunquam ante fuit, nec postea erit; et quem non audiebam, nec novi.

P. Somnium te forte fatigavit magister? - A. Etiam, fili. Audi et aliud: Vidi mortuos generare vivum, et aura vivi consumpti sunt mortui.

P. De fricatione arborum ignis natus est, consumens arbores. - A. Verum est. Audivi mortuos multa loquentes.
P. Qual é o sonho de quem está acordado? A. A esperança.

P. O que é a esperança? - A. Refresco do trabalho, evento duvidoso.

P. O que é a amizade? - A. Igualdade das almas.

P. O que é a fé? - A. A certeza de algo desconhecido e maravilhoso.

P. O que é uma maravilha? - A.

Recentemente eu vi um homem, que nunca existiu, em pé, mexendo-se e andando.

P. Como pode ser? Me explica? - A. Um reflexo na água.

P. Por que eu não entendi isso por mim mesmo, embora eu tenha visto este homem muitas vezes? - A. Porque você é um jovem de boa índole e de engenho natural, eu vou descrever para você outras maravilhas. Veja se consegue reconhecê-las por você mesmo.

P. Tentarei, mas, se eu errar, me corrija. - A. Farei como você preferir. Um desconhecido falou comigo sem língua e sem voz, alguém que não existia antes, e nem existirá depois, e que eu não ouvia e nem conhecia.

P. Talvez o sono o fatiga, professor? ${ }^{4}-\mathbf{A}$. Sim, filho. Ouça essa outra: vi mortos gerarem o vivo, e o suspiro do vivo consumir os mortos.

P. Da fricção das árvores nasce o fogo, que consome as árvores. ${ }^{5}-\mathbf{A}$. É verdade. Ouvi que os mortos falam muitas coisas.

\footnotetext{
${ }^{4}$ Uma pessoa em um sonho.

${ }^{5}$ Fogo gerado a partir de gravetos.
} 
P. Nunquam bene, nisi suspendantur in aere. - A. Vere. Vidi ignem inexstinctum pausare in aqua.

P. Silicem in aqua significare vis, reor. $-\mathbf{A}$. $\mathrm{Ut}$ reris, sic est. Vidi mortuum sedentem super vivum, et in risu mortui mortuus est vivus.

P. Hoc coci nostri norunt. - A. Norunt. Sed pone digitum super os, ne pueri hoc audiant, quid sit. Fui in venatione cum aliis, in qua si quid cepimus, nihil nobiscum portavimus; quem non potuimus capere, domum portavimus nobiscum.

P. Rusticorum est haec venatio. - A. Est. Vidi quemdam natum, antequam esset conceptus.

P. Vidisti, et forte manducasti. - A. Manducavi. Quis est, qui non est, et nomen habet et responsum dat sonanti?

P. Biblos in silva interroga. - A. Vidi hospitem currentem cum domo sua; et ille tacebat, et domus sonabat.

P. Para mihi rete, et pandam tibi. - A. Quis est, quem videre non potes, nisi clausis oculis?

P. Qui stertit, tibi ostendit illum. - A. Vidi hominem octo in manu tenentem, et de octonis subito rapuit septem, et remanserunt sex.
P. Nunca bem, a não ser que estivessem

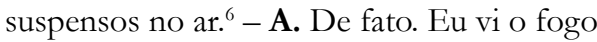
parado na água sem se apagar.

P. Você vê a sílica na água, creio. - A. É isso mesmo. Eu vi um morto sedento sobre um vivo, e no riso do morto o vivo morreu.

P. Os nossos cozinheiros sabem essa. ${ }^{7}-$ A. Sabem. Mas tampe a boca, para que as crianças não escutem o que é. Fui a uma caçada com outros, se pegamos algo, não podemos trazer conosco, e o que não podemos pegar, trouxemos conosco para casa.

P. Esta é uma caçada de grosseiros. ${ }^{8}-$

A. Sim. Eu vi algo nascido antes de ser concebido.

P. Você viu e talvez comeu. ${ }^{9}-$ A. Comi. Quem é que não existe, mas tem nome e dá uma resposta a quem chama?

P. Pergunte aos juncos da floresta. ${ }^{10}-\mathbf{A}$. Vi um hóspede correr com sua casa, e ele era silencioso e sua casa era barulhenta.

P. Me dê uma rede que eu mostro. ${ }^{11}-\mathbf{A}$. O que é que você não pode ver a não ser que feche os olhos?

P. Quem está roncando pode mostrar para você. $^{12}-\mathbf{A}$. Vi um homem que tinha oito na mão, dos oito tirou sete e permaneceram seis.

\footnotetext{
${ }^{6} \operatorname{Sinos}$

${ }^{7}$ Uma panela sobre o fogão.

${ }^{8}$ Lêndeas ou pulgas.

${ }^{9}$ Pinto no ovo.

${ }^{10}$ Eco.

${ }^{11} \mathrm{Um}$ peixe no rio.

${ }^{12} \mathrm{Um}$ sonho.
} 
P. Pueri in scholis hoc sciunt. - A. Quis est, cui, si caput abstuleris, altior surgit?

P. Vade ad lectum tuum et ibi invenies. $-\mathbf{A}$. Tres fuere; unus nusquam natus et semel mortuus. Alter semel natus, nunquam mortuus. Tertius semel natus et bis mortuus.

P. Primus aequivocus terrae; secundus Deo meo; tertius homini pauperi. - A. Dic tamen primas litteras nominum.

P. I. V. XXX. - A. Vidi feminam volantem, rostrum habentem ferreum, et corpus ligneum et caudam pennatam, mortem portantem.
P. As crianças nas escolas sabem essa. ${ }^{13}-\mathbf{A}$.

O que fica mais alto quando você retira a cabeça?

P. Vá pra sua cama e o encontrará lá. ${ }^{14}-\mathbf{A}$. Havia três: um nunca nascido e morto uma vez, o segundo nasceu uma vez e nunca morreu, o terceiro nasceu uma vez e morreu duas vezes.

P. O primeiro é equivalente à terra, o segundo ao meu Deus, o terceiro a um homem pobre. - A. Diga as primeiras letras dos nomes deles.

P. I, V, XXX. ${ }^{15}$ - A. Vi uma mulher voadora, tinha um bico de ferro, corpo de madeira e uma cauda emplumada, carregava a morte.

${ }^{13}$ A resposta desse enigma requer conhecimento de uma forma específica de contagem escolástica. Tal forma de contagem com as mãos remonta ao período clássico e sobrevive descrita em diversos manuscritos medievais. Por exemplo, em Beda, Opera de temporibus (in Halsall, 2004, p. 172): “Cum ergo dicis unum, minimum in laeua digitum inflectens, in medium palmae artum infiges. Cum dicis duo, secundum a minimo flexum, ibidem impones. Cum dicis tria, tertium similiter adflectes. Cum dicis quattuor, itidem minimum leuabis. Cum dicis quinque, secundum a minimo similiterer iges. Cum dicis sex, tertium nihilominus eleuabis, medio dumtaxat solo, qui medicus appellatur, in medium palmae fixo. Cum dicis septem, minimum solum, caeteris interim leuatis, superpalmae radicem pones. Iuxta quem cum dicis octo, medicum." "Quando, portanto, você diz 'um', dobre o dedo mínimo da mão esquerda e coloque a ponta no meio da palma. Quando você disser 'dois', dobre o segundo dedo, ao lado do menor, e coloque-o lá da mesma forma. Quando você disser 'três', dobre o terceiro dedo da mesma forma. Quando você disser 'quatro', levante o dedo mínimo novamente. Quando você disser 'cinco', levante o segundo dedo, ao lado do dedo mínimo, da mesma maneira. Quando você diz 'seis', você deve levantar o terceiro dedo, com o dedo médio [entre o terceiro dedo e o dedo mínimo - ou seja, o dedo anular], que é chamado de medicus, colocado sozinho no meio da palma. Quando você disser 'sete', coloque o dedo mínimo sozinho na palma da mão, levantando o resto. Quando você disser 'oito', coloque o dedo anelar ao lado dele.” Assim então nos explica Bayless (2004, p. 173): O sinal para o oito, então, é dobrar o dedo mínimo e o anelar para baixo; o sinal para sete é segurar apenas o dedo mínimo para baixo, e o sinal para seis é manter apenas o dedo anelar para baixo. Assim, fazer o sinal de oito - ou, como diz Alcuíno, "segure o oito na mão" - e, de repente, retirar o sinal do sete realmente produz o sinal do seis.

${ }^{14}$ Travesseiro.

15 Adão; Enoque ou Elias; Lazarus. Os numerais '.I.' e '.V.' começando as respostas se referem claramente ao lugar da letra no alfabeto: 'i’ é o A de Adão e 'v' o E de Enoque e Elias. O número 'XXX' era representado pelo Lambda grego em certos documentos medievais, e o lambda então representa o L de Lázaro. Adão, sendo feito de terra, é equivalente a ele; Enoque e Elias servem como antítipos de Cristo; e Lázaro era um homem pobre. 
P. Socia militum. - A. Quid est miles?

P. Murus imperii, timor hostium, gloriosum servitium. - A. Quid est quod est et non est?

P. Nihil. - A. Quomodo potest esse et non esse?

P. Nomine est, et re non est. - A. Quid est tacitus nuncius?

P. Quem manu teneo. - A. Quid tenes manu?

P. Epistolam meam - A. Lege feliciter, fili.
P. É amiga dos soldados. ${ }^{16}-$ A. O que é um soldado?

P. O muro do império, temor dos inimigos, serviço glorioso. - A. O que é que é e não é? P. Nada. - A. Como pode existir e não existir?

P. Existe no nome, mas não nas coisas. - A.

O que é um mensageiro calado?

P. O que tenho na mão. - A. O que você tem na mão?

P. Minha carta. - A. Boa leitura, filho.

\section{REFERÊNCIAS}

ALCUINUS. Patrologia Latina. Ed. Jacques Paul Migne. Paris, 1844. Volume MPL 101 - Colunas 0975-0980B. Disponível em: https://www.documentacatholicaomnia. eu/04z/z_0735-0804_Alcuinus_Pippini_Regalis_Et_Nobilissimi_Juvenis_Disputatio_ Cum_Albino_Scholastico_MLT.pdf.html. Acesso em: 14 set. 2020.

BAYLESS, Martha. Alcuin's Disputatio Pippini and the Early Medieval Riddle Tradition. In: HALSALL, Guy (ed.). Humour, History and Politics in Late Antiquity and the Early Middle Ages. Cambridge: Cambridge University Press, 2004, p. 157-78.

BEESON, Charles Henry. A primer of medieval Latin, an anthology of prose and poetry. Chicago: Scott Foresman and Company, 1953.

BITTERLI, Dieter. Say what I am called: the old English riddles of the Exeter Book and the Algo-Latin riddle tradition. Toronto: University of Toronto Press, 2009.

BROWN, Giles. Introduction: The Carolingian Renaissance. In: McKITTERICK, Rosamond (ed.). Carolingian culture: emulation and innovation. Cambridge University Press, 1994, p. 1-51.

BULLOUGH, Donald Auberon. Alcuin [Albinus, Flaccus] (c. 740-804), abbot of St Martin's, Tours, and royal adviser. Oxford Dictionary of National Biography (on-line). 23 Sep. 2004. Acesso em: 14 set. 2020.

COSTRINO, Artur. Alcuin's Disputatio de rhetorica: A critical edition with studies of aspects of the text, the stemma codicum, the didactic diagrams and a reinterpretation of sources for the problem of the duality of the dialogue. Doctor of Philosophy - Medieval Studies, York, University of York, 2016. Disponível em: http://etheses.whiterose.ac.uk/17792/.

${ }^{16}$ Uma flecha. 
GARRISON, Mary. The library of Alcuin's York. In: GAMESON, Richard (ed.). The Cambridge history of the book in Britain. Cambridge: Cambridge University Press, 2012, p. 633-64.

HALSALL, Guy (ed.). Humour, History and Politics in Late Antiquity and the Early Middle Ages. Cambridge: Cambridge University Press, 2004.

LAUAND, Luiz. Diálogo entre Pepino e Alcuíno. Videtur, n. 16, p. 73-82, 2002.

McKITTERICK, Rosamund (ed.). Carolingian Culture: emulation and innovation. Cambridge University Press, 1994.

ORCHARD, Andy. Alcuin's educational dispute: the riddle of teaching and the teaching of riddles. In: IRVINE, Susan; RUDOLF, Winfried (ed.). Childhood and adolescence in Anglo-Saxon literary culture. Toronto: University of Toronto Press, 2018, p. 162-201.

SCHECK, Helene. Reform and resistance, formations of female subjectivity in early medieval ecclesiastical culture. Albany: Suny Press, 2008. 